\title{
Educação em saúde e projeto integrador como alternativa para promover educação postural em crianças e adolescentes
}

Health education and integrative project as an alternative to promote postural education in children and adolescents

\section{Proyecto educativo e integrador para la salud como alternativa para promover la educación postural en niños y adolescentes}

Thays Cristine Ferro Wanderley ${ }^{1 *}$, Melissa Medeiros Maciel ${ }^{1}$, Rikelly Moura Herculano ${ }^{1}$, Wellington Tadeu Viturino Ribeiro ${ }^{1}$, Lucas Mateus dos Santos Silva ${ }^{1}$, Aline Maria Azevedo Costa ${ }^{1}$, Elionai Dias Soares $^{1}$, Felipe Lima Rebêlo ${ }^{1,2}$, Clarissa Cotrim dos Anjos ${ }^{1,2}$.

\section{RESUMO}

Objetivo: Relatar a experiência no acompanhamento e desenvolvimento de atividades educativas relacionadas à coluna vertebral e alterações posturais. Relato de experiência: $O$ estudo descreve a experiência na ação educativa desenvolvida por alunos do curso de Fisioterapia com crianças e adolescentes de uma escola particular e idade entre 12 e 17 anos. O planejamento das atividades incluiu aula em laboratório de anatomia, orientações quanto aos elementos escolhidos para a educação em saúde, construção de material didático, desenvolvimento da ação, treinamento para pesquisa e elaboração de trabalho acadêmico, e apresentação de relatório final. Os estudantes de Fisioterapia foram divididos em dez grupos e, utilizando o modelo de feira de ciências, os visitantes foram direcionados a participar das atividades oferecidas, favorecendo a construção do conhecimento. Considerações finais: Observou-se grande aceitação da população alvo, e favorecimento da construção de atividades extensionistas, construindo atividades frente à comunidade desde o início da formação acadêmica, colocando em prática o conhecimento adquirido em sala de aula, o que parece ajudar a aprimorar habilidades e competências que permeiam todo o processo de promoção da saúde.

Palavras-chave: Fisioterapia, Postura, Atenção primária à saúde.

\begin{abstract}
Objective: Report the experience in monitoring and developing educational activities related to the spine and postural changes. Experience report: The study describes the experience in the educational action developed by students of the Physiotherapy course with children and adolescents from a private school and aged between 12 and 17 years. The planning of activities included class in the anatomy laboratory, guidance on the elements chosen for health education, construction of didactic material, development of action, training for research and preparation of academic work, and presentation of a final report. Physiotherapy students were divided into ten groups and, using the science fair model, visitors were directed to participate in the activities offered, favoring the construction of knowledge. Final considerations: There was a great acceptance of the target population, and favoring the construction of extension activities, building activities in front of the community since the beginning of academic training, putting into practice the knowledge acquired in the classroom, which seems to help improve skills and competencies that permeate the entire health promotion process.
\end{abstract}

Key words: Physical therapy specialty, Posture, Primary health care.

${ }^{1}$ Centro Universitário CESMAC, Maceió - AL. *E-mail: thatyferro@hotmail.com

2 Universidade Estadual de Ciências da Saúde de Alagoas (UNCISAL), Maceió - AL. 


\section{RESUMEN}

Objetivo: Reportar la experiencia en el seguimiento y desarrollo de actividades educativas relacionadas con la columna y los cambios posturales. Informe de experiencia: El estudio describe la experiencia en la acción educativa desarrollada por alumnos del curso de Fisioterapia con niños y adolescentes de un colegio privado y con edades comprendidas entre los 12 y los 17 años. La planificación de las actividades incluyó clase en el laboratorio de anatomía, orientación sobre los elementos elegidos para la educación en salud, construcción de material didáctico, desarrollo de la acción, capacitación para la investigación y preparación de trabajos académicos y presentación de un informe final. Los estudiantes de fisioterapia se dividieron en diez grupos y, utilizando el modelo de feria de ciencias, se dirigió a los visitantes a participar en las actividades ofrecidas, favoreciendo la construcción del conocimiento. Consideraciones finales: Hubo una gran aceptación de la población objetivo, y favoreciendo la construcción de actividades de extensión, construyendo actividades frente a la comunidad desde el inicio de la formación académica, poniendo en práctica los conocimientos adquiridos en el aula, lo que parece ayudar a mejorar habilidades y competencias que permean todo el proceso de promoción de la salud.

Palabras clave: Fisioterapia, Postura, Atención primaria de salud.

\section{INTRODUÇÃO}

No Brasil, o Sistema Único de Saúde (SUS) determina que a atenção integral à saúde da criança e do adolescente implica no acesso universal e igualitário aos serviços nos três níveis da atenção buscando prevenir agravos, reduzir danos e promover qualidade de vida. Essa demanda exige a construção de ações voltadas à educação e atenção humanizada, com enfoque em mudanças de hábitos que levem aquisições por período prolongado da vida (BRASIL, 2010).

O Estatuto da Criança e do Adolescente classifica como criança a pessoa com até 12 (doze) anos de idade incompletos e adolescente entre 12 (doze) e 18 (dezoito) anos, porém, a Política Nacional de Atenção Integral à Saúde as Criança (PNAISC) considera apto a usar os serviços de pediatria do Sistema Único de Saúde (SUS) pessoas até 15 (quinze) anos de idade, sendo esse limite etário flexível, e traz o olhar sobre os agravos prevalentes na infância e doenças crônicas como eixo estratégico para orientar e qualificar as ações e serviços de saúde na atenção integral a criança (BRASIL, 1990; BRASIL, 2015).

A postura é considerada como posição ou atitude do corpo estática ou dinâmica que envolve o posicionamento alinhado do tronco e cabeça em relação ao centro de gravidade e sofre influência da base de suporte, campo visual e referências funcionais internas. Sabe-se que no período escolar, a criança e adolescente estão passando por processos maturacionais, alguns caracterizados por diversas modificações em seu crescimento e desenvolvimento, nessa situação, o alinhamento postural adequado e estruturas musculoesqueléticas equilibradas os tornam menos propensas a lesões ou deformidades (ROGGIA B, et al., 2016; BADARÓ AFV, et al., 2015; MELO RS, et al., 2011).

Em crianças e adolescentes em idade escolar a postura pode ser influenciada por fatores intrínsecos e extrínsecos incluindo as condições ambientais de estudo, peso de material escolar, uso de calçados inadequados, sedentarismo, acúmulo de tarefas acadêmicas, uso excessivo de computadores, obesidade, herança genética, fatores socioambientais, socioeconômicos emocionais e relacionados ao crescimento e desenvolvimento, que atrelados a falta de educação em saúde e desinformação podem ocasionar problemas posturais (COSTA CNC, 2019; SILVA T, et al., 2020; SILVA WS, et al., 2016).

Atividades comuns nessa faixa etária envolve o uso de smartphones, jogos virtuais e computadores e a má postura adotada nessas atividades pode levar ao desgaste da coluna vertebral, sobrecarregando-a e tornando essa população mais susceptível a lesões e alterações posturais, o que pode justificar a adoção da saúde em educação postural como eixo principal na educação em saúde escolar, possibilitando a melhora na qualidade de vida do educando, se inserida nas unidades de ensino (SAUERESSIG IB, et al., 2015; SILVA PC, 2016). 
Com o advindo da atenção primária e ruptura do modelo de assistência curativa e hospitalar as equipes multiprofissionais se mostraram essenciais na garantia da integralidade da assistência prestada pelos serviços de saúde e essa mudança também se apresentou no processo de formação do profissional fisioterapeuta que anteriormente tinha um caráter essencialmente curativo e reabilitador e veio a desenvolver competências e habilidades suficientes para a atuação em outros níveis de atenção a saúde e priorizando a integralidade no serviço (FERTONANI HP, et al., 2015; SOUZA MC, et al., 2012).

Sendo assim, considerando que crianças e adolescentes estão mais susceptíveis a desenvolver problemas posturais, por apresentarem características adaptativas do sistema locomotor em processo de crescimento, e que a implementação de ações de educação em saúde pode ser adotada como estratégia na busca a integralidade da atenção, justifica-se o desenvolvimento de atividade no modelo assistencial voltado a atenção primária, e traz o objetivo de relatar a experiência no acompanhamento e desenvolvimento de atividades educativas relacionadas a estrutura da coluna vertebral e alterações posturais, voltadas a saúde da criança e do adolescente, desenvolvidas em uma escola da região metropolitana de Alagoas.

\section{RELATO DE EXPERIÊNCIA}

Esse estudo é caracterizado como descritivo, do tipo relato de experiência, acerca da aplicação da metodologia ativa, no modelo de feira de ciências, como modalidade para desenvolver o processo de educação em saúde na criança e no adolescente, envolvendo temáticas sobre: estrutura anatômica da coluna vertebral; alterações da coluna vertebral; uso de tecnologia e disfunções posturais; postura durante estudos e Atividades da Vida Diária (AVD); carga da mochila; e técnicas posturais de avaliação e tratamento.

A ação foi desenvolvida em uma escola que assiste a crianças e adolescentes, de ambos os sexos, desde a educação infantil até o ensino médio. Para iniciar as atividades, foi realizada a apresentação da proposta como projeto integrador das disciplinas ministradas no primeiro ano do curso de Fisioterapia. A partir daí, o planejamento do projeto e da ação de educação em saúde ocorreu duas vezes ao mês, no período de agosto a novembro de 2019 , totalizando oito encontros, com duração média de uma hora. Todas as atividades de idealização foram realizadas nas instalações da instituição de ensino superior de vínculo com os discentes e docentes participantes, e a ação de educação em saúde desenvolvida nas dependências da escola.

O planejamento das atividades incluiu aula expositiva em laboratório de anatomia sobre as estruturas anatômicas da região dorsal, distribuição e orientações dos discentes quanto aos elementos escolhidos para a ação de educação em saúde, construção de material didático, desenvolvimento da ação, treinamento para pesquisa em base de dados e elaboração de trabalho acadêmico, e apresentação de relatório final do projeto integrador.

A ação educativa foi desenvolvida com as crianças e adolescentes designadas pela escola, com idade entre 12 e 17 anos, sendo convidadas as turmas de ensino fundamental e médio, assim como seus responsáveis e professores. Os estudantes de Fisioterapia foram divididos em dez grupos para otimização do trabalho e distribuição das bancadas com os temas selecionados a serem apresentados.

O modelo de feira de ciências foi a metodologia utilizada, onde os visitantes eram direcionados a participar das atividades oferecidas. Elas foram organizadas e desenvolvidas para favorecer a participação ativa e a construção do conhecimento dos participantes. As dinâmicas envolveram pintura corporal, jogos de perguntas e respostas com prendas e brindes, identificação de acertos e erros posturais por fotos de exposição, demonstração de exercícios posturais, simulação de carga da mochila e ajustes posturais no ambiente escolar. O modelo lúdico e dinâmico adotado favoreceu que os participantes fizessem parte do processo de educação em saúde, sendo observado grande aceitação e interesse.

Durante o desenvolvimento das atividades notou-se o aumento gradativo do interesse das crianças e adolescentes o que favoreceu a participação ativa dos professores e da responsável pedagógica da escola, motivando uma visita pela fisioterapeuta responsável para avaliação das estruturas e possível melhora ergonômica das estruturas em sala de aula. 


\section{DISCUSSÃO}

A dificuldade de construção do conhecimento nas ciências e na educação em saúde normalmente é oriunda da falta de aplicação prática da informação e de atividades inerentes ao interesse do aluno, e a escolha de estratégias, como a feira de ciências, pode ser uma oportunidade não convencional de promover saúde e cultura científica, despertando a criatividade e maior envolvimento dos alunos que promovem e da população assistida (DIAS FYEC, et al., 2020).

As bancadas foram divididas e planejadas pensando no desenvolvimento do conhecimento da estrutura da coluna vertebral, educação postural e ciência sobre as possibilidades de avaliação e tratamento de alterações posturais, dessas bancadas, quatro atenderam a temática central de desenvolver o conhecimento sobre a anatomia da coluna vertebral, incluindo a estrutura óssea, articular e muscular. Para despertar o interesse pelo aprendizado foram utilizados modelos anatômicos sintéticos, maquetes e pintura corporal. Nas bancadas seguintes os participantes eram direcionados a colocar em prática o conhecimento sobre a coluna vertebral e postura em atividades cotidianas, incluindo o uso de celulares, computador, carregar objetos, usa de mochilas escolares e sentar-se na cadeira escolar.

Hiperlordose lombar, retificação cervical, protrusão da cabeça e escoliose são alterações posturais de alta prevalência em crianças e adolescentes e o conhecimento do corpo e das possibilidades de avaliação e tratamento para essas disfunções pode tornar a intervenção necessária precoce e maiores serão as possibilidades de sucesso, com grandes chances de prevenir progressões e evitar agravos na fase adulta (BADARÓ AFV, et al, 2015; PRETO LSR, et al., 2015).

Pesquisa de caráter epidemiológica e populacional, desenvolvida no Brasil, com 1.546 alunos descreveu alta prevalência de dor nas costas (64\%) e da adoção de postura inadequada ao carregar a mochila, escrever na mesa escolar, usar o computador e pegar objetos no chão, entendendo que quando a posição corporal é inadequada o sistema musculoesquelético fica comprometido (NOLL M, et al., 2017).

Ao serem instruídos sobre as estruturas anatômicas da coluna vertebral entendemos que seria facilitado o processo de aprendizado sobre as alterações posturais da coluna vertebral, sendo assim, foram desenvolvidas três estruturas com informações que davam ênfase às alterações da coluna vertebral, métodos de avaliação postural e demonstração de técnicas fisioterapêuticas e, em situações de queixas álgicas, os docentes solicitaram avaliação da fisioterapeuta responsável que realizou o encaminhamento, quando necessário, para a Clínica Escola de uma Instituição de Ensino Superior responsável pelo projeto integrador.

As avaliações posturais e orientações para os escolares podem responder de maneira positiva, pois ajuda a diminuir diversos riscos de desvios ou de dor nas costas. Esta é uma medida que deve ser tomada para melhor educação postural escolar, com orientação adequada, realizada por fisioterapeuta, orientando e acompanhando o progresso de cada criança ou adolescente, quanto a adequação postural (ZERO JR e PORTES LA, 2013).

Considerando as orientações para as atividades da vida diária das crianças e adolescentes, foram selecionadas três bancadas para abordar as alterações posturais associadas ao uso da tecnologia, postura durante as atividades diárias e escolares e a carga na mochila. Para esses temas, abordamos metodologias ativas com jogos e simulação, através de fotografias dos docentes do curso de Fisioterapia e demonstrações, explanando a maneira correta e incorreta de manter-se sentado, pegar objetos pesados e carregar a mochila escolar.

Estudos trazem o ambiente escolar como de risco para o desenvolvimento de alterações posturais e lombalgia e dentre os fatores de influência estão a carga excessiva da mochila, mobiliários inadequados em sua estrutura, sedentarismo, posturas estáticas prolongadas e movimentos repetitivos, alertando sobre a necessidade de programas de promoção da saúde para aumentar a conscientização e reduzir os riscos nesse ambiente (SOUZA ES, et al., 2020; ADAMATTI C, et al., 2020).

Para abordar sobre o uso da mochila escolar realizou-se simulações com livros didáticos, balança e modelos diversos de mochilas, como o objetivo de relacionar o peso corporal com a carga diária da mochila 
e informar da relação ideal. Percebemos que a maioria dos escolares carregavam, um excesso de peso e utilizam o apoio somente em um dos membros superiores, podendo levar ao maior risco de alteração postural. Os professores relataram que, apesar do colégio realizar um rodízio de disciplinas que utilizam o mesmo livro didático e disponibilizar um espaço para guardar os materiais, não há a utilização adequada dele, o que pode apontar a necessidade e educação em saúde e esclarecimentos.

Para verificar a presença de dor na coluna vertebral e os fatores associados Saes MO e Soares MCF (2017) realizaram um estudo com 619 adolescentes escolares entre 10 e 17 anos utilizando o Questionário Nórdico de Sintomas Osteomusculares e análise de outras variáveis como dor durante o percurso de ida e volta da escola e forma de transporte do material escolar. Os autores identificaram alta prevalência de dor vertebral entre os adolescentes $(45,2 \%)$ além da associação com o peso da mochila e a forma como ela era transportada e recomendam que revejam a orientação de carga até $10 \%$ do peso corporal, principalmente no sexo feminino.

Essa orientação de reduzir o limite da carga em mochilas de escolares também foi feita por Ries LG, et al. (2012), em estudo que avaliou a postura de 50 escolares com idades entre 8 e 14 anos em situação de carga variável da mochila, peso habitual, $10 \%, 15 \%$ e $20 \%$ do Peso Corporal (PC) na qual foi identificada a anteriorização de cabeça mesma em cargas inferiores a $10 \%$ do peso corporal que pode ser direcionada ao estresse físico e compensações na cinemática da coluna vertebral em todos os planos de movimentos.

Porém, a carga da mochila não é a única responsável por alterações posturais em escolares, Sedrez JA, et al. (2015) identificou uma prevalência de $79 \%$ de alterações posturais nos planos frontal e sagital, em 59 crianças e adolescentes, e encontrou associação não somente com o transporte do meio escolar, mas também com baixa atividade física, tempo de sono superior a 10 horas e postura sentada inadequada, essa sobrecarga na região dorsal pode inclusive ocasionar maior pressão nos discos intervertebrais, levando a propensão de desgaste e disfunção dessa estrutura anatômica (VIEIRA A, et al., 2015). Além disso, atualmente, a manutenção da postura em sedestação, com o uso por tempo prolongado de tecnologias como televisão, tablet, smartphone e computador integram outro grupo de fatores de risco para alterações posturais em crianças e adolescentes, principalmente na curvatura cifótica da região torácica (SEDREZ JA, et al., 2015).

A associação entre a postura sentada para assistir televisão e o aumento da prevalência de escoliose, avaliada pelo teste de Adam, foi identificada em estudo com 954 crianças e familiares. A permanência da posição estática, com pouca movimentação ou movimentação inadequada, leva a sobrecarga da coluna vertebral e, quando somado a esse hábito diário de assistir televisão, leva ao aumento na probabilidade de desvios posturais (CIACCIA MCC, et al., 2017).

Entende-se que a escola é um ambiente de grande relevância para a promoção da saúde, estimulando a melhora nas condições de saúde e qualidade de vida, e atrelado ao uso de metodologias ativas, pode aumentar o potencial da ação educativa, levando a melhoria no processo saúde/doença (ANJOS CC, et al., 2017).

Dessa forma, observou-se uma grande aceitação e participação por parte das crianças, adolescentes, responsáveis e professores, além da demonstração de interesse em mudança de hábitos e adequações estruturais o que favoreceu a percepção da importância da inserção da Fisioterapia no ambiente escolar para ampliar a visão sobre orientação postural e desenvolvimento musculoesquelético. Assim como, a construção de atividades frente à comunidade desde o início da formação acadêmica, colocando em prática 0 conhecimento adquirido em sala de aula, pode ajudar a aprimorar habilidades e competências, incluindo a capacidade de desenvolver o raciocínio clínico, trabalhar em equipe e lidar com os fatores emocionais, inerentes às relações humanas e que permeiam todo o processo de promoção da saúde.

\section{REFERÊNCIAS}

1. ADAMATTI C, et al. Alterações posturais na adolescência. Perspectiva: Ciência e Saúde, 2020; 5 (1): 1-11.

2. ANJOS CC, et al. Ações de Fisioterapia voltadas à saúde da criança em uma escola de ensino fundamental em Maceió, Alagoas. Rev. Bra. Edu. Saúde, 2017: 7 (1): 49-54. 
3. BADARÓ AFV, et al. Investigação da postura corporal de escolares em estudos brasileiros. Fisioter. Pesqui., 2015; 22 (2): 197-204.

4. BRASIL. Lei no 8.069, de 13 de julho de 1990. Dispõe sobre o Estatuto da Criança e do Adolescente e dá outras providências. Brasília: Diário Oficial da União. 1990. Disponível em: <http://www.planalto.gov.br/ccivil_03/leis//8069.htm>. Acesso em: 9 dez. 2020.

5. BRASIL. Ministério da Saúde. Secretaria de Atenção à Saúde. Departamento de Ações Programáticas Estratégicas. Linha de cuidado para a atenção integral à saúde de crianças, adolescentes e suas famílias em situação de violências: orientação para gestores e profissionais de saúde. Brasília: Ministério da Saúde, 2010. Disponível em: <https://bvsms.saude.gov.br/bvs/publicacoes/linha_cuidado_criancas_familias_violencias.pdf>. Acesso em: 9 dez. 2020.

6. BRASIL. Portaria ํㅜ 1.130, de 5 de agosto de 2015. Institui a Política Nacional de Atenção Integral à Saúde da Criança (PNAISC) no âmbito do Sistema Único de Saúde (SUS). Brasília: Diário Oficial da União, 2015. Disponível em: <http://bvsms.saude.gov.br/bvs/saudelegis/gm/2015/prt1130_05_08_2015.html>. Acesso em: 9 dez. 2020.

7. CIACCIA MCC, et al. Prevalence of scoliosis in public elementary school students. Rev. Paul Pediatr., $2017 ; 35$ (2): 191-198.

8. DIAS FYEC, et al. O papel da Feira de Ciências como estratégia motivadora para o ensino de Botânica na educação básica. Hoehnea, 2020; 47, e552019.

9. FERTONANI HP, et al. Modelo assistencial em saúde: conceitos e desafios para a atenção básica brasileira. Ciênc. Saúde Colet., 2015; 20 (6): 1969-1878.

10. COSTA CNC. Análise das diferenças posturais e comparação da flexibilidade muscular em crianças negras, brancas e orientais de 9 a 15 anos de idade. Dissertação (Mestrado em Ciências) - Faculdade de Medicina. Universidade de São Paulo, São Paulo, 2019; 73 p.

11. MELO RS, et al. Avaliação postural da coluna vertebral em crianças e adolescentes com deficiência auditiva. Arquivos Int. Otorrinolaringol. (Impr.), 2011; 15 (2): 195-202.

12. NOLL M, et al. Dor nas costas e hábitos comportamentais de estudantes do ensino médio: um estudo comparativo entre duas regiões do Brasil. Rev. Bras. Reumatol., 2017; 57 (5): 495-499.

13. PRETO LSR, et al. Análise por Fotogrametria da Postura e Fatores de Risco Associados em Crianças e Adolescentes Escolarizados. Rev. Enf. Ref., 2015; 4 (7): 31-40.

14. RIES LG, et al. Os efeitos de diferentes pesos de mochilas no alinhamento postural de crianças em idade escolar. Motricidade, 2012; 8 (4): 87-95.

15. ROGGIA B, et al. Postura e equilíbrio corporal de escolares de oito a doze anos com e sem respiração oral. CoDAS, 2016; 28 (4): 395-402.

16. SAES MO, SOARES MCF. Fatores associados a dor na coluna vertebral em adolescentes de escolas públicas de um município do extremo sul do Brasil. Rev. salud pública, 2017; 19 (1): 105-111.

17. SAUERESSIG IB, et al. Prevalência de dor musculoesquelética em adolescentes e sua associação com o uso de dispositivos eletrônicos. Rev. dor, 2015; 16 (2): 129-135, jun.

18. SEDREZ JA, et al. Fatores de risco associados a alterações posturais estruturais da coluna vertebral em crianças e adolescentes. Rev. paul. pediatr., 2015; 33 (1): 72-81.

19. SILVA PC. Alterações posturais em crianças frequentadoras de escolas municipais da cidade de Salvador - Bahia. Diálogos \& Ciências, 2016; 16 (37): 16-30.

20. SILVA T, et al. Qualidade de vida e prevalência de dor na região cervical em acadêmicos. Revista Eletrônica Acervo Saúde, 2020; 39: 1-9.

21. SILVA WS, et al. Análise da postura em escolares de 8 a 14 anos de Maceió-Al: um estudo transversal descritivo. Arquivos em Movimento, 2016; 12 (2): 43-53.

22. SOUZA ES, et al. Perfil da sintomatologia dolorosa da coluna vertebral em estudantes de graduação em um município do Maranhão. Revista Eletrônica Acervo Científico, 2020; 16: 1-8.

23. SOUZA MC, et al., Integralidade na atenção à saúde: um olhar da Equipe de Saúde da Família sobre a fisioterapia. Mundo Saúde, 2012; 36 (3): 452-460.

24. VIEIRA A, et al. Efeitos de um Programa de Educação Postural para escolares do terceiro ano do Ensino Fundamental de uma escola estadual de Porto Alegre (RS). Fisioter. Pesqui., 2015; 22 (3): 239-245.

25. ZERO JR, ORTES LA. Orientação postural e análise do peso da mochila de alunos do primeiro e segundo ano do Ensino Fundamental no Município de Leme/SP. Rev. Bras. Fisiologia do Exercício, 2013; 12 (5): 287-291. 